\title{
Incomplete Success of Angioinhibitor Therapy in Cancer: Estimation of Contribution of Pro-angiogenic Activity of Patient Thyroid Hormone
}

Paul J Davis ${ }^{1,2 *}$, Murat Yalcin ${ }^{1}$, Hung-Yun Lin ${ }^{3}$, Heng-Yuan Tang ${ }^{1}$, Aleck Hercbergs ${ }^{4}$, John T. Leith ${ }^{5}$, Faith B Davis ${ }^{1}$, Mary K Luidens ${ }^{2}$ and Shaker A Mousa ${ }^{1}$

${ }^{1}$ Pharmaceutical Research Institute, Albany College of Pharmacy and Health Sciences, Rensselaer, NY, USA

${ }^{2}$ Department of Medicine, Albany Medical College, Albany, NY, USA

${ }^{3}$ Graduate Institute of Cancer Biology and Drug Discovery, College of Medical Science and Technology, Taipei Medical University, Taipei, Taiwan

${ }^{4}$ Department of Radiation Oncology, the Cleveland Clinic, Cleveland, $\mathrm{OH}, \mathrm{USA}$

${ }^{5}$ Rhode Island Nuclear Research Center, Providence, RI, USA

\begin{abstract}
Despite the obvious promise of the strategy, pharmaceutical angioinhibition has had variable success in clinical cancer management. Thyroid hormone is a potent pro-angiogenic factor. Endogenous circulating levels of proangiogenic thyroid hormone in cancer patients treated with anti-angiogenic drugs may contribute to host resistance to angioinhibition and explain, at least in part, the variable cancer chemotherapeutic responses obtained with anti-angiogenic agents. The chick chorioallantoic membrane (CAM) angiogenesis assay accepts human tumor xenografts and is a system in which individual patient blood samples can be tested in xenograft vasculature for anti-angiogenic content-including thyroid hormone-in the presence of angioinhibitory drug dose escalation. The assay may also be used to screen individual patient tumor biopsy xenografts for susceptibility to angioinhibition.
\end{abstract}

Keywords: Thyroxine; Angiogenesis; Chick chorioallantoic membrane (CAM) assay; Integrin $\alpha v \beta 3$

\section{Introduction}

Angiogenesis inhibitor therapy directed at one or several vascular growth factors or their receptors in cancer has had variable success, despite the hypervascularity of many tumors. Treatment success that is only partial may reflect redundancy of vascular growth factors in cancer cells, tolerance for hypovascularity and hypoxia of certain tumors, resistance mutations in growth factor receptor genes or the presence of effective circulating endogenous pro-angiogenic substances in the cancer patient. Estrogen and progesterone are examples of steroids that are pro-angiogenic in their target tissues $[1,2]$ and target tissue cancers.

By multiple molecular mechanisms, thyroid hormone-Lthyroxine $\left(\mathrm{T}_{4}\right)$ and 3,5,3'-triiodo-L-thyronine $\left(\mathrm{T}_{3}\right)$ [3,4]-and other thyroid hormone agonist analogues $[5,6]$ have been shown to be pro-angiogenic. Pharmacologic elimination of this action of thyroid hormone at a cell surface receptor for the hormone can in days reduce the vascularity of human tumor xenografts by $40-50 \%$ [7-9] and contribute to shrinkage of tumor volume. Thyroid hormone can also support proliferation of cancer cells [10-12], so that change in tumor size reflects several mechanisms when activity of the hormone is reduced at the tumor site. The pro-angiogenic activity of $\mathrm{T}_{4}$ and $\mathrm{T}_{3}$ is initiated at a cell surface receptor for the hormones on a structural protein of the plasma membrane, integrin $\alpha v \beta 3$ [13]. The original description of this receptor was in an angiogenic model system, the chick chorioallantoic membrane (CAM) assay [13]. The integrin has multiple functions that relate to extracellular matrix proteins [14], to growth factor receptors on the cell surface [15] and, within the cell, to cell structure [16] and specific gene transcription [17]. The existence of the iodothyronine receptor on the integrin has broadened our appreciation of the contributions that a small molecule ligand-integrin interaction can make to regulation of cancer-relevant angiogenesis $[18,19]$ and to gene transcription involved in cancer cell survival pathways $[8,19,20]$. This iodothyronine receptor is subject to specific blockade with tetraiodothyroacetic acid (tetrac), a derivative of $\mathrm{T}_{4}$, and a nanoparticulate covalent conjugate of tetrac [19]. These two agents rapidly express in tumor xenografts the loss of vascularity to which we referred [7-9].

That cancer in the setting of hypothyroidism, spontaneous or induced, behaves differently than in euthyroidism is suggested by clinical studies of glioblastoma [21], breast cancer [22] and, apparently, of renal cell carcinoma [23-25]. In each of these settings, desirable clinical behavior accompanies decreased circulating levels of thyroid hormone and this may in part reflect actions of thyroid hormone on angiogenesis. It is important to emphasize that clinical hypothyroidism is not advocated here to affect tumor cell behavior desirably, since medically-induced subclinical hypothyroidism (asymptomatic elevation of serum thyrotropin [TSH]) has been effective in importantly arresting progression and increasing overall survival in glioblastoma patients [21]. In a second cancer-relevant context, it should also be pointed out that thyroid hormone is anti-apoptotic [26,27], supporting survival of the cancer cell and its angiogenic activity. The hormone has been shown to inhibit apoptosis induced in vitro by specific agents such as resveratrol [27] and ceramide (HY Lin: unpublished).

We propose that certain host circulating nonpeptide hormonesthe model here is thyroid hormone-that are pro-angiogenic may limit in selected patients or specific clinical settings the effectiveness of anticancer therapy that is focused exclusively on angiogenesis.

*Corresponding author: Paul J Davis, M.D., Pharmaceutical Research Institute Albany College of Pharmacy and Health Sciences, One Discovery Drive, Rensselaer, NY 12144, USA, Tel: 518428 7848; Fax: 518694 7567; E-mail: pdavis.ordwayst@gamil.com

Received November 01, 2013; Accepted December 26, 2013; Published December 30, 2013

Citation: Davis PJ, Yalcin M, Lin HY, Tang HY, Hercbergs A, et al. (2013) Incomplete Success of Angioinhibitor Therapy in Cancer: Estimation of Contribution of Proangiogenic Activity of Patient Thyroid Hormone. J Cancer Sci Ther 5: 441-445. doi:10.4172/1948-5956.1000238

Copyright: $\odot 2013$ Davis PJ, et al. This is an open-access article distributed under the terms of the Creative Commons Attribution License, which permits unrestricted use, distribution, and reproduction in any medium, provided the original author and source are credited. 
Citation: Davis PJ, Yalcin M, Lin HY, Tang HY, Hercbergs A, et al. (2013) Incomplete Success of Angioinhibitor Therapy in Cancer: Estimation of Contribution of Pro-angiogenic Activity of Patient Thyroid Hormone. J Cancer Sci Ther 5: 441-445. doi:10.4172/1948-5956.1000238

Estrogen [28,29], as suggested above, and gonadotropins [30] are other possible examples of nonpeptide and peptide hormones, respectively, that may modulate tumor-relevant angiogenesis. In the case of thyroid hormone, elevated circulating free $\mathrm{T}_{4}\left(\mathrm{FT}_{4}\right)$ may be encountered transiently or longer in the nonthyroidal illness (NTI) syndrome or in patients receiving high-dose ${\mathrm{L}-\mathrm{T}_{4}}_{4}$ replacement. The NTI syndrome includes low serum $\mathrm{T}_{3}$ concentration and suppressed TSH [31]. Thus, in the setting of NTI, reduced circulating levels of $\mathrm{T}_{3}$ and TSH are not germane to angiogenesis, but $\mathrm{FT}_{4}$ is supportive. The issue may also be raised for consideration that endogenous free $T_{4}$ concentrations in the upper quartile of the reference range in euthyroid patients may support angiogenesis. In the state of subclinical hypothyroidism, $\mathrm{FT}_{4}$ is normal and circulating $\mathrm{TSH}$, another peptide hormone, is by definition elevated and conceivably could be a pro-angiogenic factor in nonthyroidal tumor tissue [32]. The regulatory input of thyroid hormone into angiogenesis is discussed in more detail below.

The question is how to estimate the contribution of any endogenous host growth factors to suboptimal efficacy of angioinhibiton therapy. The CAM assay accepts human cancer xenografts in which tumor growth properties are readily detected [12] and tumor vascularity quantitated $[4,13]$. This assay is accessible and inexpensive. It is a vehicle in which responsiveness of standard tumor cell lines to antiangiogenic treatment agents has been assessed. As discussed below, the assay is a possible test system in which individual patients' plasma or serum may be screened for pro-angiogenic properties in the presence of xenografted tumor and specific anti-angiogenic biological or chemical agents.

\section{Pro-angiogenic Properties of Thyroid Hormone}

Studies in myocardium by Tomanek et al. [3,33] established that $\mathrm{T}_{4}$ and a thyroid hormone analogue, diiodothyropropionic acid (DITPA) [34], stimulated coronary arteriolar growth. Subsequently and prior to the description of the existence of the thyroid hormone receptor on $\alpha v \beta 3$, we showed that the hormone was pro-angiogenic in the CAM model [4] by a cell surface-based mechanism. Components of the mechanism of angiogenesis included transcription of the basic fibroblast growth factor $(b F G F)$ gene and release of the gene product into the medium of the CAM. Thyroid hormone also induces microtubule formation by human dermal microvascular endothelial cells [5] and stimulates migration of endothelial cells towards a vitronectin cue in the modified Boyden chamber apparatus (SA Mousa: unpublished observations). Evidence subsequently developed in the CAM assay suggested that the hormone may also influence the activities of vascular endothelial growth factor (VEGF) [35] and platelet-derived growth factor (PDGF) (SA Mousa: unpublished). That is, a pharmacologic inhibitor of thyroid hormone action at $\alpha v \beta 3$ decreased pro-angiogenic actions of VEGF and PDGF in the CAM. The mechanism is thought to involve crosstalk between integrin $\alpha v \beta 3$ and the adjacent receptors for VEGF, PDGF and bFGF on the cell surface. It is also clear that thyroid hormone can modulate certain non-angiogenic functions of the plasma membrane receptors for epidermal growth factor (EGF) [36] and insulin-like growth factor-1 (IGF-1) [37]. But EGF and IGF$1[38,39]$ do have pro-angiogenic properties and it will be important to determine whether crosstalk between receptors for these growth factors and the iodothyronine receptor on $\alpha v \beta 3$ exists. The complexity of the relationship between iodothyronines and IGF-1 and the latter's receptor (IGF1R) is demonstrated in studies of rodent skeletal myoblasts. In these cells, thyroid hormone and IGF-1 individually stimulate muscle cell proliferation and hexose uptake, but the hormone blocks action of IGF-1 on both functions [37]. Thus, thorough studies of these agents, individually and together, are required in a standard angiogenesis model, such as the CAM, to determine whether these growth factors work to block therapeutic angioinhibition.

We have confirmed that certain agonist thyroid hormone analogues such as DITPA [6] and the non-iodinated synthetic hormone analogue, GC-1 [40], are pro-angiogenic in the CAM assay. DITPA had previously been shown to increase myocardial VEGF and bFGF in the nonischemic rat heart [34]. Pro-angiogenic qualities of thyroid hormone have also been shown experimentally in the ischemic limb [41] and in wound-healing [42,43] (Figure 1).

\section{Thyroid Hormone Analogues and Angiogenesis Associated with Cancer Xenografts}

In xenografts in the euthyroid nude mouse of human renal cell carcinoma [7], follicular thyroid cancer [44], medullary carcinoma of the thyroid [8] and pancreatic cancer [9], we have induced frank and rapid decrease in vascularity of the grafts with a pharmacologic inhibitor of actions of $\mathrm{T}_{4}$ and $\mathrm{T}_{3}$ at their receptor on $\alpha v \beta 3$. This angiogenesis inhibitor is unmodified or nanoparticulate tetrac [19]. The latter involves covalent binding of tetrac to a nanoparticle to prevent cellular uptake of the thyroid hormone analogue and limit drug activity to the extracellular domain of the integrin $a v \beta 3$. The proangiogenic activity of thyroid hormone in the CAM assay can also be blocked with monoclonal antibody to $\alpha v \beta 3$ [19].

It should be emphasized that integrin $\alpha v \beta 3$ is expressed generously by rapidly-dividing blood vessel cells and by cancer cells. Quiescent



Control



bFGF



T4

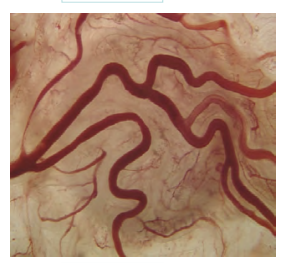

VEGF

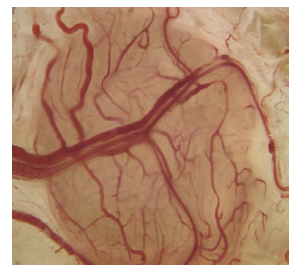

T4 NP

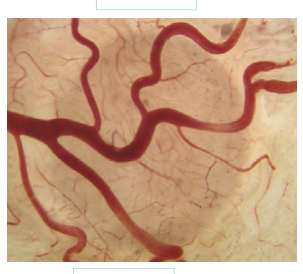

DITPA
Figure 1: Photographic images of chick chorioallantoic membrane (CAM) assays of angiogenic activity of L-thyroxine ( $T_{4}$ ), nanoparticulate $T_{4}$ (T4 NP) [19], bFGF, VEGF and diiodothyropionic acid (DITPA) [6], a thyroid hormone analogue. Control is phosphate-buffered saline. The methodology is described in Ref. 4. T4 NP is a formulation in which the hormone is covalently-bound via a linker to a nanoparticule (poly[lactic-co-glycolic acid]); the nanoparticle precludes cellular uptake of the hormone and limits activity of $T_{4}$ to a receptor on the cell exterior (integrin $\alpha \mathrm{v} \beta 3$ ) that regulates angiogenesis [13] and a variety of other cell functions [19]. The integrin is generously expressed by rapidlydividing endothelial cells, as in this assay, and by tumor cells and osteoclasts [19]. Like $\mathrm{T}_{4}$ and T4 NP, DITPA initiates its proangiogenic activity at av $\beta 3$. Vascular branch points in the CAM assay may be quantified $[4,6]$ to enable statistical comparisons of activities of biologic fluids, of different pharmacologic agents and of endogenous growth factors. Among the latter are bFGF and VEGF, shown here. We propose the use of the assay for measurement of proangiogenic activity in human blood that may oppose cancer chemotherapy that is primarily anti-angiogenic. 
nonmalignant cells and platelets express the activated integrin only in small amounts [19].

\section{Testing for the Contribution of Endogenous Thyroid Hormone to Cancer-relevant Angiogenesis}

The CAM system not only indexes tumor-related angiogenesis, but permits spherical tumor growth of the implanted cells. Spherical growth is important to the development of a hypoxic cell population (JT Leith: unpublished observations) that resembles that of tumor behavior clinically and that supports radioresistance. Aggressive xenograft behavior in the CAM model includes metastasis to the chick embryo in the system [12]. These qualities of the assay are desirable in a cancer modeling system.

Vessel branch points are readily quantitated in the CAM system by analysis with standardized software of digitized images [4], permitting statistical evaluation of possible pro-angiogenic ('anti-angioinhibition') effects of patient sera in presence and absence of chemotherapeutic agent(s) [4,13]. The fertilized eggs are commercially available. The model can be exploited in multiple strategies, as discussed below.

In grafts of human lung cancer to the CAM exposed to tetrac, tumor vascularity is promptly reduced [7-9]. The CAM is euthyroid because of the contribution of maternal thyroid hormone to the egg and the maturation of the embryonic pituitary-thyroid axis by the time of application of tetrac to the model at 10-12 days of development in ovo. The results obtained with tetrac are consistent with loss of proangiogenic activity of thyroid hormone. But, as mentioned above, tetrac also may affect the angiogenic activities of VEGF [35] and bFGF $[4,35]$, in the absence of $\mathrm{T}_{4}$ and $\mathrm{T}_{3}$. The endpoint in studies of the proangiogenic action of thyroid hormone is histologic examination of the grafts for degree of vascularity.

It may also be desirable to study the behavior of established tumor grafts in a CAM rendered hypothyroid or to determine the interplay of pro-angiogenic properties of thyroid hormone and anti-angiogenic pharmaceuticals in the hypothyroid CAM system. We suggest that the 'euthyroid' CAM model containing an established human cancer implant may be rendered acutely hypothyroid as needed by exchange transfusion of embryonic serum treated with anion exchange resin [45]. Acute reductions in circulating endogenous thyroid hormone are required because established hypothyroidism will result in arrested tumor cell proliferation and decreased release of vascular growth factors; these factors will reduce detectability of anti-angiogenic activity of an added pharmaceutical or biological.

Specific strategies for use of the CAM in the context of antiangiogenesis therapy include the following: (1) estimation of relapsed patient pro-angiogenic ('anti-angioinhibitory') activity in the absence of tumor grafts in the CAM, when first-line anti-angiogenic treatment has failed or when anti-angiogenesis is being considered as second-line therapy and specific treatment agent activity can be measured in the CAM in the presence of patient serum samples; (2) estimation of host pro-angiogenic activity in standard tumor cell line grafts in the CAM that are relevant to specific, relapsed patients-the assays also test specific treatment agents; (3) estimation of host pro-angiogenic activity in the presence of biopsied cell grafts from the patient in the CAM, with and without specific anti-angiogenic/chemo-therapeutic agent(s). Each of these approaches personalizes care.

In the clinical setting, several other paradigms can be considered for evaluating the potential contribution of endogenous (host) thyroid hormone to the impact of therapeutic angioinhibition. These potential approaches include a search for correlations between circulating free $\mathrm{T}_{4}$ levels and anti-tumor effectiveness of anti-angiogenic agents. In the absence of anti-angiogenic therapy, it may also be useful to attempt to quantitate the contribution of endogenous thyroid hormone to tumor vasculature. This strategy would involve estimation histologically of the ratio of tumor vascularity: tumor cellularity in biopsies from hypothyroid vs. euthyroid cancer patients at the same institution in protocols such as that for the breast cancer population characterized at MD Anderson Cancer Center by Cristofanilli and co-workers [22] and the glioblastoma patient cohort at the Cleveland Clinic in which biochemical hypothyroidism was medically induced [21]. Blood free $\mathrm{T}_{4}$ levels at the times of biopsy should be included in the analysis.

\section{Discussion}

The pro-angiogenic properties of thyroid hormone have been satisfactorily demonstrated in several model systems [3,4,46-48]. That endogenous circulating thyroid hormone may clinically oppose the anti-angiogenic chemotherapy of cancer in patients has not been considered. We have proposed that the levels of hormone, as $\mathrm{T}_{4} /$ free $\mathrm{T}_{4}$, may be sufficiently high in certain cancer patients to blunt the effect of therapy specifically directed at one or several vascular growth factors and thus limit the anti-tumor effectiveness of the therapeutic agent. We cite $\mathrm{T}_{4}$ specifically here because (1) the NTI syndrome which cancer patients may exhibit is associated with low serum $\mathrm{T}_{3}$ concentrations and (2) the effect of $\mathrm{T}_{3}$ on tumor cell proliferation is less than that of thyroxine. We speculate that levels of $\mathrm{T}_{4}$ in the upper quartile of the normal range or that are sufficient to depress circulating TSH ('subclinical hyperthyroidism') may be adequate to oppose pharmacologic angioinhibition by multiple mechanisms. Only prospective, controlled testing of medical induction of chemical hypothyroidism-raising the serum TSH to the upper limit of the reference range or above, but avoiding clinical symptoms of hypothyroidism-will satisfactorily address this issue. This has been done systematically in the setting of endstage glioblastoma multiforme, a highly vascular tumor, with reasonable success [21]. Coincident hypothyroidism appears to have changed the course of breast cancer in one large clinical experience [22], as noted above, reducing the aggressiveness of the disease. The latter study was not designed to segregate possible effects of decreased thyroid function on tumor cells or on tumor-associated vasculature, nor was the recent observation that incidental induction of hypothyroidism by tyrosine kinase inhibitors as a side effect is an important contributor to effect on another vascular tumor, renal cell carcinoma [24].

The receptor for thyroid hormone and hormone analogues on integrin $\alpha v \beta 3$ permits thyroid hormone to affect a number of regulatory factors in the process of angiogenesis, including function of multiple vascular growth factors [19], their receptors and angiogenic chemokines [49]. Small molecules such as bradykinin and angiotensin II are pro-angiogenic and their activity in this regard is modulated by the integrin (SA Mousa: unpublished observations). As noted above, the receptor on $\alpha v \beta 3$ influences endothelial cell motility (SA Mousa: unpublished observations) and vascular microtubule formation [5]. A prototypical inhibitor of thyroid hormone at the receptor on the integrin is Nanotetrac and this experimental nanoparticulate formulation of tetrac is effective as an anti-angiogenic substance in multiple model assays [19].

The second component of the pituitary-thyroid hormonal axis, 
Citation: Davis PJ, Yalcin M, Lin HY, Tang HY, Hercbergs A, et al. (2013) Incomplete Success of Angioinhibitor Therapy in Cancer: Estimation of Contribution of Pro-angiogenic Activity of Patient Thyroid Hormone. J Cancer Sci Ther 5: 441-445. doi:10.4172/1948-5956.1000238

polypeptide thyrotropin (TSH) also supports vascular endothelial growth factor (VEGF) expression in thyroid cancer [50] and normal thyroid tissue [51]. Interestingly, a TSH receptor also exists on normal human dermal vascular endothelial cells; the receptor promotes VEGF expression in such cells in response to TSH [32]. Because primary hypothyroidism, accompanied by increases in serum TSH, appears to improve the clinical courses of certain important tumorsglioblastoma, breast, kidney, as mentioned above-it is unlikely that elevated circulating TSH is an important patient factor in reduced effectiveness of anti-angiogenic treatment of cancers.

In the current paper we propose the experimental use of the CAM assay in several paradigms to estimate anti-angiogenic properties of blood samples from cancer patients. The CAM model system also lends itself to screening of grafted tumors whose cells are harvested from individual patients for susceptibility to available anti-angiogenic clinical agents. Such studies would reflect the intrinsic susceptibility or resistance to angioinhibition of tumors established in the CAM from individual subjects, independent of circulating host factors such as thyroid hormone. But patient tumors established in the CAM system may be re-exposed to donor (host) serum or plasma in the model in the presence and absence of chemotherapeutic agents proposed for use in an example of personalization of tumor management.

In an extension of studies by the present authors of the effects of thyroid hormone analogues on radiosensitivity of tumor cells established in cell culture [52,53], Leith has demonstrated that assessment of tumor graft radiosensitivity is also feasible in the CAM model (JT Leith: unpublished observations).

The advantages of the CAM model include its history of use to estimate pro- and anti-angiogenic treatment agents in industry and academic centers, its standardization as a quantitative model of angiogenesis, its ready tolerance and support of human tumor cells and the low cost from commercial sources of 10 day-old chick embryos (fertilized eggs). The CAM summates actions of endogenous pro-angiogenic factors, including thyroid hormone, providing more information than individual assays for one or several possible antiangioinhibitory factors. The system requires as many as 6-8 eggs per experimental variable, is thus not a high-throughput system, and of course has not been validated for the purposes proposed here.

\section{References}

1. Fraser HM, Duncan WC (2009) SRB Reproduction, Fertility and Development Award Lecture 2008. Regulation and manipulation of angiogenesis in the ovary and endometrium. Reprod Fertil Dev 21: 377-392.

2. Rogers PA, Donoghue JF, Walter LM, Girling JE (2009) Endometria angiogenesis, vascular maturation, and lymphangiogenesis. Reprod Sci 16: 147-151.

3. Tomanek RJ, Doty MK, Sandra A (1998) Early coronary angiogenesis in response to thyroxine: growth characteristics and upregulation of basic fibroblast growth factor. Circ Res 82: 587-593.

4. Davis FB, Mousa SA, O'Connor L, Mohamed S, Lin HY, et al. (2004) Proangiogenic action of thyroid hormone is fibroblast growth factor-dependent and is initiated at the cell surface. Circ Res 94: 1500-1506.

5. Mousa SA, Davis FB, Mohamed S, Davis PJ, Feng X (2006) Pro-angiogenesis action of thyroid hormone and analogs in a three-dimensional in vitro microvascular endothelial sprouting model. Int Angiol 25: 407-413.

6. Mousa SA, O'Connor L, Davis FB, Davis PJ (2006) Proangiogenesis action of the thyroid hormone analog 3,5-diiodothyropropionic acid (DITPA) is initiated at the cell surface and is integrin mediated. Endocrinology 147: 1602-1607.

7. Yalcin M, Bharali DJ, Lansing L, Dyskin E, Mousa SS, et al. (2009) Tetraidothyroacetic acid (tetrac) and tetrac nanoparticles inhibit growth of human renal cell carcinoma xenografts. Anticancer Res 29: 3825-3831.
8. Yalcin M, Dyskin E, Lansing L, Bharali DJ, Mousa SS, et al (2010) Tetraiodothyroacetic acid (tetrac) and nanoparticulate tetrac arrest growth of medullary carcinoma of the thyroid. J Clin Endocrinol Metab 95: 1972-1980.

9. Yalcin M, Lin HY, Sudha T, Bharali DJ, Meng R, et al. (2013) Response of human pancreatic cancer cell xenografts to tetraiodothyroacetic acid nanoparticles. Horm Cancer 4: 176-185

10. Lin HY, Tang HY, Shih A, Keating T, Cao G, et al. (2007) Thyroid hormone is a MAPK-dependent growth factor for thyroid cancer cells and is anti-apoptotic. Steroids 72: 180-187.

11. Lin HY, Sun M, Tang HY, Lin C, Luidens MK, et al. (2009) L-Thyroxine vs. 3,5,3'-triiodo-L-thyronine and cell proliferation: activation of mitogen-activated protein kinase and phosphatidylinositol 3-kinase. Am J Physiol Cell Physiol 296: C980-C991.

12. Mousa SA, Yalcin M, Bharali DJ, Meng R, Tang HY, et al. (2012) Tetraiodothyroacetic acid and its nanoformulation inhibit thyroid hormone stimulation of non-small cell lung cancer cells in vitro and its growth in xenografts. Lung Cancer 76: 39-45.

13. Bergh JJ, Lin HY, Lansing L, Mohamed SN, Davis FB, et al. (2005) Integrin alphaVbeta3 contains a cell surface receptor site for thyroid hormone that is linked to activation of mitogen-activated protein kinase and induction of angiogenesis. Endocrinology 146: 2864-2871.

14. Plow EF, Haas TA, Zhang L, Loftus J, Smith JW (2000) Ligand binding to integrins. J Biol Chem 275: 21785-21788.

15. Schneller M, Vuori K, Ruoslahti E (1997) Alphavbeta3 integrin associates with activated insulin and PDGFbeta receptors and potentiates the biological activity of PDGF. EMBO J 16: 5600-5607.

16. Zou W, Teitelbaum SL (2010) Integrins, growth factors, and the osteoclast cytoskeleton. Ann N Y Acad Sci 1192: 27-31.

17. Roth P, Silginer M, Goodman SL, Hasenbach K, Thies S, et al. (2013) Integrin control of the transforming growth factor- $\beta$ pathway in glioblastoma. Brain 136 : 564-576.

18. Pinto M, Soares $P$, Ribatti $D$ (2011) Thyroid hormone as a regulator of tumor induced angiogenesis. Cancer Lett 301: 119-126.

19. Davis PJ, Davis FB, Mousa SA, Luidens MK, Lin HY (2011) Membrane receptor for thyroid hormone: physiologic and pharmacologic implications. Annu Rev Pharmacol Toxicol 51: 99-115.

20. Glinskii AB, Glinsky GV, Lin HY, Tang HY, Sun M, et al. (2009) Modification of survival pathway gene expression in human breast cancer cells by tetraiodothyroacetic acid (tetrac). Cell Cycle 8: 3554-3562.

21. Hercbergs AA, Goyal LK, Suh JH, Lee S, Reddy CA, et al. (2003) Propylthiouracil-induced chemical hypothyroidism with high-dose tamoxifen prolongs survival in recurrent high grade glioma: a phase I/II study. Anticancer Res 23: 617-626.

22. Cristofanilli M, Yamamura Y, Kau SW, Bevers T, Strom S, et al. (2005) Thyroid hormone and breast carcinoma. Primary hypothyroidism is associated with a reduced incidence of primary breast carcinoma. Cancer 103: 1122-1128.

23. Hercbergs AH, Ashur-Fabian O, Garfield D (2010) Thyroid hormones and cancer: clinical studies of hypothyroidism in oncology. Curr Opin Endocrinol Diabetes Obes 17: 432-436.

24. Schmidinger M, Vogl UM, Bojic M, Lamm W, Heinzl H, et al. (2011) Hypothyroidism in patients with renal cell carcinoma: blessing or curse? Cancer 117: 534-544.

25. Riesenbeck LM, Bierer S, Hoffmeister I, Köpke T, Papavassilis P, et al. (2011) Hypothyroidism correlates with a better prognosis in metastatic renal cancer patients treated with sorafenib or sunitinib. World J Urol 29: 807-813.

26. Lin HY, Tang HY, Shih A, Keating T, Cao G, et al. (2007) Thyroid hormone is a MAPK-dependent growth factor for thyroid cancer cells and is anti-apoptotic Steroids 72: 180-187.

27. Lin HY, Tang HY, Keating T, Wu YH, Shih A, et al. (2008) Resveratrol is proapoptotic and thyroid hormone is anti-apoptotic in glioma cells: both actions are integrin and ERK mediated. Carcinogenesis 29: 62-69.

28. Zou LB, Shi S, Zhang RJ, Wang TT, Tan YJ, et al. (2013) Aquaporin-1 plays a crucial role in estrogen-induced tubulogenesis of vascular endothelial cells. J Clin Endocrinol Metab 98: E672-682. 
Citation: Davis PJ, Yalcin M, Lin HY, Tang HY, Hercbergs A, et al. (2013) Incomplete Success of Angioinhibitor Therapy in Cancer: Estimation of Contribution of Pro-angiogenic Activity of Patient Thyroid Hormone. J Cancer Sci Ther 5: 441-445. doi:10.4172/1948-5956.1000238

29. Lee JE, Chung KW, Han W, Kim SW, Kim SW, et al. (2004) Effect of estrogen, tamoxifen and epidermal growth factor on the transcriptional regulation of vascular endothelial growth factor in breast cancer cells. Anticancer Res 24 3961-3964.

30. Sapoznik S, Cohen B, Tzuman Y, Meir G, Ben-Dor S, et al. (2009) Gonadotropin-regulated lymphangiogenesis in ovarian cancer is mediated by LEDGF-induced expression of VEGF-C. Cancer Res 69: 9306-9314.

31. Farwell AP (2013) Nonthyroidal illness syndrome. Curr Opin Endocrinol Diabetes Obes 20: 478-484.

32. Balzan S, Del Carratore R, Nicolini G, Beffy P, Lubrano V, et al. (2012) Proangiogenic effect of TSH in human microvascular endothelial cells through its membrane receptor. J Clin Endocrinol Metab 97: 1763-1770.

33. Chilian WM, Wangler RD, Peters KG, Tomanek RJ, Marcus ML (1985) Thyroxine-induced left ventricular hypertrophy in the rat. Anatomical and physiological evidence for angiogenesis. Circ Res 57: 591-598.

34. Wang X, Zheng W, Christensen LP, Tomanek RJ (2003) DITPA stimulates bFGF, VEGF, angiopoietin, and Tie-2 and facilitates coronary arteriolar growth Am J Physiol Heart Circ Physiol 284: H613-618.

35. Mousa SA, Bergh JJ, Dier E, Rebbaa A, O'Connor LJ, et al. (2008) Tetraiodothyro-acetic acid, a small molecule integrin ligand, blocks angiogenesis induced by vascular endothelial growth factor and basic fibroblast growth factor. Angiogenesis 11: 183-190.

36. Shih A, Zhang S, Cao HJ, Tang HY, Davis FB, et al. (2004) Disparate effects of thyroid hormone on actions of epidermal growth factor and transforming growth factor-? are mediated by 3',5'-cyclic adenosine monophosphate-dependent protein kinase II. Endocrinology 145: 1708-1717.

37. Incerpi S, Lin HY, D Vito P, Fiore AM, Luly P, et al. (2013) Thyroid hormone inhibition of IGF-1-mediated glucose uptake and proliferation in L6 myoblasts: new roles for integrin $\alpha \vee \beta 3$.

38. Haleagrahara N, Chakravarthi $S$, Mathews $L$ (2011) Insulin like growth factor-1 (IGF-1) causes overproduction of IL-8, an angiogenic cytokine and stimulates neovascularization in isoproterenol-induced myocardial infarction in rats. Int $\mathrm{J}$ Mol Sci 12: 8562-8574.

39. Piecewicz SM, Pandey A, Roy B, Xiang SH, Zetter BR, et al. (2012) Insulin-like growth factors promote vasculogenesis in embryonic stem cells. PLoS One 7: e32191.

40. Mousa SA, O'Connor LJ, Bergh JJ, Davis FB, Scanlan TS, et al. (2005) The proangiogenic action of thyroid hormone analogue $\mathrm{GC}-1$ is initiated at an integrin. J Cardiovasc Pharmacol 46: 356-360.

41. El-Eter E, Rebbaa H, Alkayali A, Mousa SA (2007) Role of thyroid hormone analogues in angiogenesis and the development of collaterals in the rabbit hind limb ischemia model. J Thromb Thrombolysis 5: 375

42. Safer JD, Crawford TM, Holick MF (2004) A role for thyroid hormone in wound healing through keratin gene expression. Endocrinology 145: 2357-2361.

43. Safer JD (2013) Thyroid hormone and wound healing. J Thyroid Res 2013 : 124538

44. Yalcin M, Bharali DJ, Dyskin E, Dier E, Lansing L, et al. (2010) Tetraiodothyroacetic acid and tetraiodothyroacetic acid nanoparticle effectively inhibit the growth of human follicular thyroid cell carcinoma. Thyroid 20: 281 286.

45. Cao Z, West C, Norton-Wenzel CS, Rej R, Davis FB, et al. (2009) Effects of resin or charcoal treatment on fetal bovine serum and bovine calf serum. Endocr Res 34: 101-108.

46. Chen J, Ortmeier SB, Savinova OV, Nareddy VB, Beyer AJ, et al. (2012) Thyroid hormone induces sprouting angiogenesis in adult heart of hypothyroid mice through the PDGF-Akt pathway. J Cell Mol Med 16: 2726-2735.

47. Zhang L, Cooper-Kuhn CM, Nannmark U, Blomgren K, Kuhn HG (2010) Stimulatory effects of thyroid hormone on brain angiogenesis in vivo and in vitro. J Cereb Blood Flow Metab 30: 323-335.

48. Jiang JY, Miyabayashi K, Nottola SA, Umezu M, Cecconi S, et al. (2008) Thyroxine treatment stimulated ovarian follicular angiogenesis in immature hypothyroid rats. Histol Histopathol 23: 1387-1398.

49. Davis PJ, Glinsky GV, Lin HY, Incerpi S, Davis FB, et al. (2013) Molecular mechanisms of actions of formulations of the thyroid hormone analogue, tetrac, on the inflammatory response. Endocr Res 38: 112-118.

50. Hoffmann S, Hofbauer LC, Scharrenbach V Wunderlich A, Hassan I, et al. (2004) Thyrotropin (TSH)-induced production of vascular endothelial growth factor in thyroid cancer cells in vitro: evaluation of TSH signal transduction and of angiogenesis-stimulating growth factors. J Clin Endocrinol Metab 89: 61396145.

51. Klein M, Brunaud L, Muresan M, Barbé F, Marie B, et al. (2006) Recombinant human thyrotropin stimulates thyroid angiogenesis in vivo. Thyroid 16: 531 536.

52. Hercbergs A, Davis PJ, Davis FB, Ciesielski MJ, Leith JT (2009) Radiosensitization of GL261 glioma cells by tetraiodothyroacetic acid (tetrac). Cell Cycle 8: 2586-2591

53. Hercbergs AH, Lin HY, Davis FB, Davis PJ, Leith JT (2011) Radiosensitization and production of DNA double-strand breaks in U87MG brain tumor cells induced by tetraiodothyroacetic acid (tetrac). Cell Cycle 10: 352-357. 